\title{
Seroepidemiological Study of Malaria in A Former High Endemic Region of Greece
}

\author{
Dionysia Theocharidou*1, Efstratios Maltezos², Theodoros Constantinidis3 and Anna Papa ${ }^{4}$ \\ ${ }^{1}$ Microbiology specialist, Greece \\ ${ }^{2}$ Professor of Internal Medicine, Medical School, Democritus University of Thrace, Greece
}

${ }^{3}$ Professor of the Laboratory of Hygiene and Environmental Protection, Medical School, Democritus University of Thrace, Greece

${ }^{4}$ Professor of Microbiology, Medical School, Aristotle University of Thessaloniki, Greece

*Corresponding author: Dionysia Theocharidou, Microbiology specialist, Greece

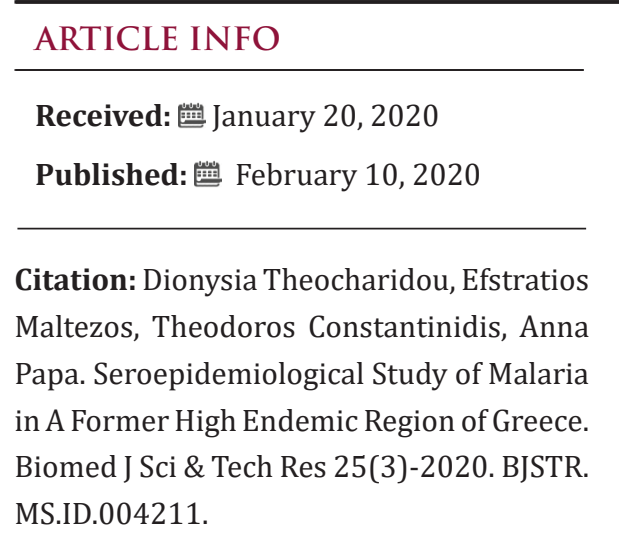

Abbreviations: RDTS: Rapid Diagnostic Tests, IFT: Immunofluorescence Test, ELISA: Enzyme-Linked Immunosorbent Assays, WHO: World Health Organization

\begin{abstract}
After malaria elimination in Greece, in 1974, a few sporadic indigenous malaria cases have been reported, up until 2009 that the country experienced the threat of disease re-emergence due to sociological and environmental factors. Northern Greece, a highly malarious place in the past, reported autochthonous cases attributed to $P$. vivax, a parasite that can cause asymptomatic infections. In the present study 347 residents of Drama province (median age: 53 years, age range: $11-94$ years, $62 \%$ women), Northern Greece, were screened, by serology, smear microscopy and Rapid Diagnostic Tests (RDTs), to evaluate and detect asymptomatic malaria cases. No seropositive individuals were identified by ELISA and blood smear microscopy was negative for all participants. ELISA borderline results were furtherly tested with RDT (sensitivity for $P$. vivax infection: 97.4\%, specificity for P. vivax infection: $99.4 \%$ ) and proved to be negative for active plasmodium parasitaemia. It is concluded that currently, Drama population remains malaria naive and as a result unable to serve as a reservoir and infect mosquitoes, contributing to the malaria transmission cycle.
\end{abstract}

Keywords: Greece Malaria Re-Emergence; Greece Asymptomatic Malaria; Greece Indigenous Malaria Transmission; Asymptomatic Malaria Diagnostics.

\section{Short Communication}

Greece had been a malaria-endemic country from prehistoric times to 1974, with northern Greece (Macedonia and Thrace) being characterized as highly malarious place. Disease elimination through deployed anti-malaria measures was corroborated after an extensive seroepidemiological study, carried out in Northern Greece, which observed no antibody titers among young people aged $<20$ years, data indicative of disease transmission absence during the past 20 years Drakeley et al. [1]. In the post-elimination setting, the vast majority of malaria reported cases was introduced, apart from sporadic reports of indigenous cases. From 1994 to 1995 four presumably autochthonous malaria cases were diagnosed by blood smear microscopy in Evros Province, Northern Greece. As a result, a seroepidemiological study conducted in 1997- 1999 at the same place showed that $0,8 \%$ of the individuals tested were seropositive Kampen, et al. [2]. It was in 2009 that autochthonous cases commenced re-emerging in Greece, leading to the formation of specific malarious hot spots (such as Evrotas region) in the country Danis et al. [3].

\section{Aim of the Study}

Among 107 reported autochthonous malaria cases during the period 2009-2018, 16 (15\%) have as suspected transmission site the northern Greek mainland. The vast majority of autochthonous malaria cases was attributed to P. vivax infection. P. vivax can cause asymptomatic or cryptic infections, which play a significant role in disease transmission even in low transmission settings. These infections may present low parasitaimia, which may be undetectable with smear microscopy, and persistently high antibody titers Baum et al. [4]. Based on prementioned sporadic indigenous cases the present study is an attempt to evaluate and detect asymptomatic 
malaria cases in Drama Province, Northern Greece. It is a $3500 \mathrm{~km}^{2}$ region, counting 100,000 citizens, neighbouring to Bulgaria, and was characterized as highly malarious in the past.

\section{Materials and Methods}

To achieve this goal, we evaluated the serological status of 366 blood samples, drawn from 347 healthy individuals (median age 53 years, age range 11- 94 years old, 62\% women), residents of Drama province. After providing their full written participation consent, they filled in a questionnaire on basic epidemiological data, travel history, work and leisure time activities. The study was approved by the Ethics Committee of Democritus University of Thrace. Blood was collected and stored at $-20^{\circ} \mathrm{C}$, and serological status of 347 individuals was defined by a commercially available ELISA Antibody test (MALAB, DIA.PRO, MILANO, ITALY), which uses recombinant P. falciparum and P. vivax antigens, and exceeds antibody detection even against P. malariae and P. ovale due to crossreactivity (95\% sensitivity and $98 \%$ specificity).

Borderline results were furtherly tested with direct immunochromatographic Standard Diagnostics BiolineTM rapid test, which uses monoclonal antibodies against pHRP-II for P. falciparum detection, and against pLDH for the detection of all human-related Plasmodium species. This rapid diagnostic test has recently been evaluated in comparison with microscopy and PCR for the diagnosis of $P$. vivax cases in Greece and has proven to have excellent sensitivity ( $97.4 \%$ and $95.6 \%$ respectively) and specificity (99.4\% and 100\% respectively). Tseroni, et al. [5]. In addition, Giemsa stained thin and thick peripheral blood smears were microscopically examined by two different clinical microbiologists reporting the results in different records.

\section{Results}

(Table 1) represents the studied population data. No individuals tested proved to be seropositive for malaria antibodies. All microscopic blood smears proved to be negative for malaria parasites. However, 12 (3,5\%) of people tested showed borderline results and were furtherly examined through RDTs, which proved to be negative for plasmodium infection. In addition, 335 (96.5\%) participants were seronegative.

Table 1: Epidemiological data of studied population, Drama, Northern Greece, $(n=347$ participants).

\begin{tabular}{|c|c|c|}
\hline \multicolumn{3}{|c|}{ Characteristic (\%) } \\
\hline Gender $n=347$ & \multicolumn{2}{|c|}{$\begin{array}{c}\text { Females: } 215 \text { (62) } \\
\text { Males: } 132(38)\end{array}$} \\
\hline Age (years) N=306 & $\begin{array}{l}\text { Median age: } 53 \\
\text { Range: } 11-94\end{array}$ & $\begin{array}{l}\text { Age Groups } \\
\begin{array}{l}\text { 0- } \quad 20: 23(7.5) \\
\text { 21- 40: } 72(23.5) \\
\text { 41- 60: } 96(31.4) \\
\text { 61- 80: } 94(30.7) \\
>80: 21(6.9)\end{array}\end{array}$ \\
\hline \multirow[t]{2}{*}{ Travel History N=243 } & Yes: 85 (35), Malaria endemic country: 0 & \\
\hline & No: $158(65)$ & \\
\hline \multirow[t]{2}{*}{ Residency N=335 } & Urban: 155 (46.3) & \\
\hline & Rural: 180 (53.7) & \\
\hline \multirow[t]{2}{*}{ Occupation $N=309$} & Agropastoral: 122 (39.5) & \\
\hline & Office: 187 (60.5) & \\
\hline \multirow[t]{2}{*}{ Malaria Abs N=347 } & Negative: 335 (96.5) & \\
\hline & Borderline: 12 (3.5) & \\
\hline
\end{tabular}

\section{Discussion}

Seroepidemiological studies using antibodies measurement against Plasmodium parasites with Immunofluorescence Test (IFAT) or Enzyme-Linked Immunosorbent Assays (ELISA) can provide reliable information for the effectiveness of control measurements and for changes on malaria transmission. In addition, subclinical malaria cases caused by all human-related plasmodium species could play a substantial role in disease transmission dynamics.
Given the fact that Drama province used to be a malarious, swampy region in the past, residents suffered from high malaria prevalence due to P. falciparum, P. vivax and P. malariae. Especially during World War II, when anti-malarial measures were disrupted, Northern Greece suffered from heavy outbreaks every summer. It should be noted that malaria cases have been diagnosed during the period 1994-1999 in northern Greece (Evros province) among individuals with no previous travel history Kampen et al. [6]. Massive efflux of 
immigrants from malaria endemic countries in the last years, the presence of plasmodium-transmitting vectors, and the favourable climatic and landscape conditions reinforced $P$. vivax indigenous transmission in Greece since 2009.

The parasite $P$. vivax is more capable to re-emerge in such an environment through the formation of hypnozoites and its ability to secure its survival in temperate climates Piperaki et al. [7]. Our study in the province of Drama failed to detect any seropositivity for malaria, indicating that the inhabitants of this area of northern Greece are currently malaria naive without asymptomatic infections and without significant risk to infect the local competent for malaria Anopheles species. This study is the second attempt to identify occult malaria cases after disease re-emergence threat in Greece. In 2015 both residents of hot-spots and of low-risk regions have been tested revealing no seropositive individuals Piperaki et al. [8]. It is of note, that among 306 participants, who provided information concerning date of birth, 21 (6.9\%) were elder individuals (>80 years old), some of whom clearly recalled, during questionnaire interviews, a wide malaria distribution in the region and reported specific epidemiological data on disease transmission in the past.

As a result, some of those participants may have contracted the disease during childhood. In fact, 2 of them mentioned quinine intake during childhood, unable to recall whether it was administered on a preventive or a therapeutic basis. However, even those individuals were seronegative, indicating that antibody titers decrease in a few months after successful treatment Pinto, et al. [9]. Both peripheral blood smear testing and antibody detection showed total compliance for diagnosing seronegative samples. The microscopic examination didn't detect intracellular parasites at any of the samples furtherly tested with RDTs, which proved to be negative. Given the fact, that even post 2009 the majority of malaria reported cases are introduced, travel history was recorded for most participants ( $\mathrm{n}=$ 243), and 85 (35\%) reported travelling at 1- 12 different countries, however, none of the mentioned destinations was malaria endemic according to World Health Organization (WHO).

\section{Conclusion}

Our study indicates that the indigenous population is malaria naive and unable to serve as a reservoir for further malaria

\section{ISSN: 2574-1241}

DOI: $10.26717 /$ BJSTR.2020.25.004211

Dionysia Theocharidou. Biomed J Sci \& Tech Res

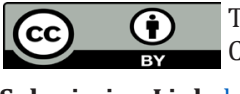

This work is licensed under Creative

Commons Attribution 4.0 License

Submission Link: https://biomedres.us/submit-manuscript.php transmission. However, the humanitarian crisis leading to massive population movementalong with favourable entomological, climatic and landscape conditions have led to sporadic autochthonous cases. As a result, in such an unstable setting, high clinical awareness is required both for rapid and accurate diagnosis and for active detection of asymptomatic cases or patients that do not refer to health authorities.

\section{Conflict of Interest}

All authors declare that they have no conflict of interest. The study was approved by the Ethics Committee of DUTH. All participants provided their full written consent and were able to withdraw their participation at all stages of the study. For underaged individuals, written consent was provided by their parents.

\section{References}

1. Drakeley C, Cook J (2009) Chapter 5 Potential contribution of seroepidemiological analysis for monitoring malaria control and elimination: historical and current perspectives. Advances in Parasitology 69: 299352.

2. Kampen H, Proft J, Etti S, Maltezos E, Pagonaki M, et al. (2003) Individual cases of autochthonous malaria in Evros Province, northern Greece: entomological aspects. Parasitology Research 89(4): 252-258.

3. Danis K, Baka A, Lenglet A, Bortel W, Van, et al. (2011) Autochthonous Plasmodium vivax malaria in Greece. Eurosurveillance 16(42): 1-5.

4. Baum E, Sattabongkot J, Sirichaisinthop J, Kiattibutr K, Davies, et al. (2015) Submicroscopic and asymptomatic Plasmodium falciparum and Plasmodium vivax infections are common in western Thailand molecular and serological evidence. Malaria Journal 14(1): 95.

5. Tseroni M, Pervanidou D, Tserkezou P, Rachiotis G, Pinaka O, et al. (2015) Field Application of SD Bioline Malaria Ag Pf/Pan Rapid Diagnostic Test for Malaria in Greece. PLOS ONE 10(3): e0120367.

6. Kampen H, Maltezos Æ E, Pagonaki Æ M (2002) Individual cases of autochthonous malaria in Evros Province, northern Greece: serological aspects. Parasitology Research 88(3): 261-266.

7. Piperaki ET, Daikos GL (2016) Malaria in Europe: emerging threat or minor nuisance? Clinical Microbiology and Infection 22(6): 487-493.

8. Piperaki E, Mavrouli M, Tseroni M, Routsias J, Kallimani A, et al. (2015) Assessment of Antibody Responses in Local and Immigrant Residents of Areas with Autochthonous Malaria Transmission in Greece. 93(March 2012): 153-158.

9. Pinto AY, Das N, Ventura AMR (2001) Resposta de anticorpos IgG anti-Plasmodium vivax em crianças expostas à malária, antes e após tratamento específico. Jornal de Pediatria 77(4).

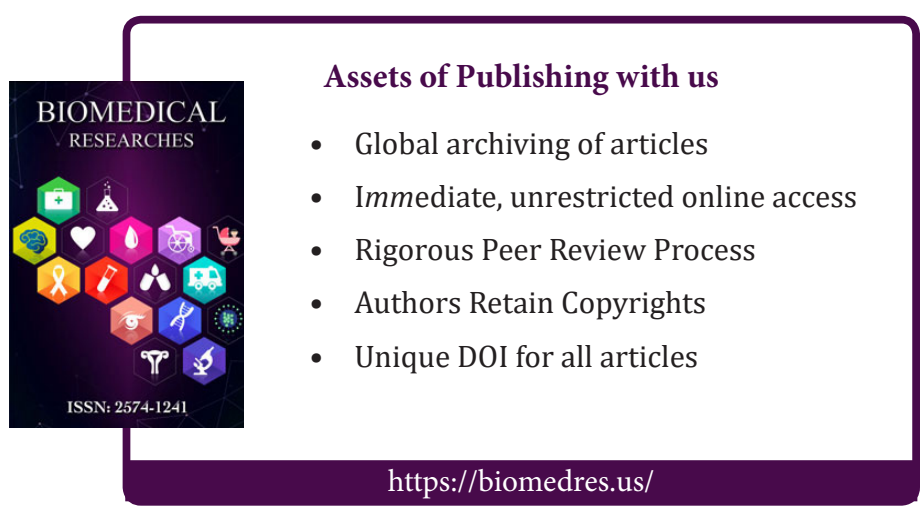

